\title{
Cosmic Superstrings: Dynamics and Cusps
}

\author{
William Nelson ${ }^{1}$ \\ King's College London, Strand, London, WC2R 2LS, U. K. \\ Institute of Gravitation and the Cosmos, Penn State University, State College, PA 16801, U. S. A.
}

\begin{abstract}
Whilst standard field theoretic Cosmic Strings cannot end, Cosmic Superstrings can form three string junctions, at which each string ends. This opens up a new class of possible boundary conditions for such strings and we show that, at least when the junctions are close together, a string ending of two such junctions will generically have cusps. Cusps are of particular interest as they are strong emitters of radiation (both gravitational and particle) and hence are possible observables. The detection of cusps from Cosmic Superstrings between junctions would be a rare observational window into the realm of String theory and Brane inflation models.
\end{abstract}

Keywords: Cosmic Superstrings, Cusps, Brane Cosmology

PACS: $11.10 . \mathrm{Lm}, 11.25 . \mathrm{Wx}, 98.80 . \mathrm{Cq}$

\section{INTRODUCTION}

Standard 2 field theoretic Cosmic Strings form during symmetry breaking phase transitions, in which the first nontrivial homotopy group of the symmetry breaking group is order 1. Or equivalently, when the vacuum manifold is topologically $S^{1}$ (see for example Ref. [3] for an introduction to Cosmic Strings and Cosmic Superstrings). As they are topological objects, they cannot end and thus form either loops or infinitely long strings (i.e. they stretch across the horizon). For scales much larger than the thickness of the string (the 'thin string' approximation), the dynamics of Cosmic Strings can be approximated by the Nambu-Goto action,

$$
S=-\mu \int \mathrm{d} \tau \mathrm{d} \sigma \sqrt{-\gamma}
$$

where $\mu$ is the tension of the string, $(\tau, \sigma)$ are two world sheet coordinates that are respectively time-like and space-like and $\gamma$ is the determinant of $\gamma_{\alpha \beta}$, the induced world sheet metric given by,

$$
\gamma_{\alpha \beta}=x_{, \alpha}^{\mu} x_{, \beta}^{v} g_{\mu v}
$$

where $g_{\mu \nu}$ is the space-time metric, and $\alpha, \beta, \ldots=(1,2)=(\tau, \sigma)$, whilst $\mu, \nu, \ldots=(0,1,2,3)$ label the space-time coordinates. Working in Minkowski space-time (as we will do through out), we can fix the world sheet gauge freedoms due to the world sheet reparameterization invariance, by making $\gamma_{\alpha \beta}$ conformal. This gives the conditions,

$$
\dot{x}^{2}+x^{\prime 2}=0, \quad \dot{x} \cdot x^{\prime}=0,
$$

where the dot refers to a derivative with respect to $\tau$ and the dash a derivative with respect to $\sigma$. Finally we can impose the static gauge conditions, $\tau=t=x^{0}$, which fixes all the residual freedom. The equation of motion given by the variation of Eq. (1) with respect to $x$, is just the standard wave equation, which has solutions in terms of arbitrary right and left movers: $\mathbf{a}(t-\sigma)$ and $\mathbf{b}(t+\sigma)$,

$$
\mathbf{x}=\frac{1}{2}[\mathbf{a}(t-\sigma)+\mathbf{b}(t+\sigma)],
$$

where $x=(t, \mathbf{x})$. In terms of these left and right movers, the gauge constraints, Eq. (3) become especially simple: $\left|\mathbf{a}^{\prime}\right|=\left|\mathbf{b}^{\prime}\right|=1$, where here the dash refers to the total derivative. Thus the solutions to the equation of motion can be

\footnotetext{
${ }^{1}$ This work was done in collaboration with Anne-Christine Davis, Senthooran Rajamanoharan, and Mairi Sakellariadou [1].

2 i.e. Albelian Cosmic Strings, Non-Albelian versions can also have junctions and are a useful way of modeling the dynamics of Cosmic Superstrings. See Ref. [2] for some recent work in this area.
} 
visualized by considering $\mathbf{a}^{\prime}$ and $\mathbf{b}^{\prime}$ as curves on the unit sphere. For the case of closed Cosmic Strings, we have that $\sigma=\sigma+L$ (again working in Minkowski space-time), where $L$ is the coordinate length of the string. If we take the zero momentum frame i.e. the frame moving with the center of mass of the loop of Cosmic String, then both $\mathbf{a}^{\prime}$ and $\mathbf{b}^{\prime}$ are also periodic in $L$. Moreover, one can show that they satisfy the 'center of mass' conditions,

$$
\frac{1}{L} \int_{0}^{L} \mathbf{a}^{\prime} \mathrm{d} \sigma=\frac{1}{L} \int_{0}^{L} \mathbf{b}^{\prime} \mathrm{d} \sigma=0 .
$$

Thus we can visualize the solutions to the equations of motion, that also satisfy the gauge constraints, as close curves on the unit sphere, that average to zero according to Eqs. (5). Because the two curves $\mathbf{a}^{\prime}$ and $\mathbf{b}^{\prime}$ are independent, one can easily see that they will generically intersec 3 . At intersection, we have $\mathbf{a}^{\prime}=\mathbf{b}^{\prime}$ and from Eq. (4) we see that such points have velocity given by,

$$
\dot{\mathbf{x}}=\mathbf{a}^{\prime}=\mathbf{b}^{\prime},
$$

which, by our gauge constraints, implies that such points have unit velocity. These luminous points are referred to as cusps. See, for example, Ref. [4] for a more detailed discussion of cusps on Cosmic Strings and topological defects in general. Naturally one cannot have a massive object moving at the speed of light, instead what this tells us, is that the thin string approximation breaks down. Despite this, general arguments [5] and field theory simulations [6] have shown that cusps still produce vast amounts of both gravitational and particle radiation, making them candidates for possible observation (see for example Refs. [7, 8]).

The purpose of this paper is to emphasize what changes (and what remains the same) when we consider Cosmic Superstrings.

\section{COSMIC SUPERSTRINGS}

Cosmic Superstrings come in two flavors: fundamental F-strings and D-strings (either D1-branes or D $p$-branes, with $p-1$ transverse directions aligned along the compactified dimensions). They are characterized by the presence of a world sheet electromagnetic field, $A_{\alpha}$, for which the ends of F-strings are sources [9]. This allows Cosmic Superstrings to form three string junctions, in which an F-string ends on a D-string, forming a bound state FD-string, which carries the conserved flux of $A_{\alpha}[10]$. The interest in Cosmic Superstrings comes from the fact that they would be copiously produced at the end of brane inflation [11] and hence would be a probe of the early, stringy, universe. They also would naturally have small tensions, which would help explain the increasingly tight observational upper bounds that have been found for the tensions of one dimensional defects. In the following we will look at the presence of cusps on Cosmic Superstrings as a possible way of distinguishing Cosmic Superstrings from their field theoretic cousins. Of particular interest is the fact that cusps naturally radiate energy into both gravitational waves and particles. In standard Cosmic Strings, these particles are all possible excitations of the symmetry group from which the string arose. In Cosmic Superstrings however, all possible excitations available in string theory might be excited e.g. Dilatons [12], Kluza-Klein modes, moduli fields, SUSY particles etc. Thus cusps on Cosmic Superstrings are a possible (if remote) direct test of string theory, as well as a consequence of early universe brane inflation models.

\subsection{A single Cosmic Superstring}

The starting point for this discussion on Cosmic Superstrings is the DBI action 4 , which for a single string is

$$
S=-\mu \int \mathrm{d} \tau \mathrm{d} \sigma \sqrt{-\left|\gamma_{\alpha \beta}+\lambda F_{\alpha \beta}\right|},
$$

where, $F_{\alpha \beta}=\partial_{\alpha} A_{\beta}-\partial_{\beta} A_{\alpha}$ is the world sheet electromagnetic field tensor and $\lambda=2 \pi \alpha^{\prime}$, with $\alpha^{\prime}$ the Regge slope of String Theory. It can be shown [13] that the gauge freedoms are again fixed by the constraints given in Eq. (3) and that

\footnotetext{
${ }^{3}$ the word 'generically' is important here, since it is possible to construct two closed curves on the unit sphere, with appropriate averages, that do not intersect. However such cases are rather special and there is a strong relationship between the two curves. Hence they are not considered to be generic.

${ }^{4}$ See, for example, [9] for a derivation of this action.
} 


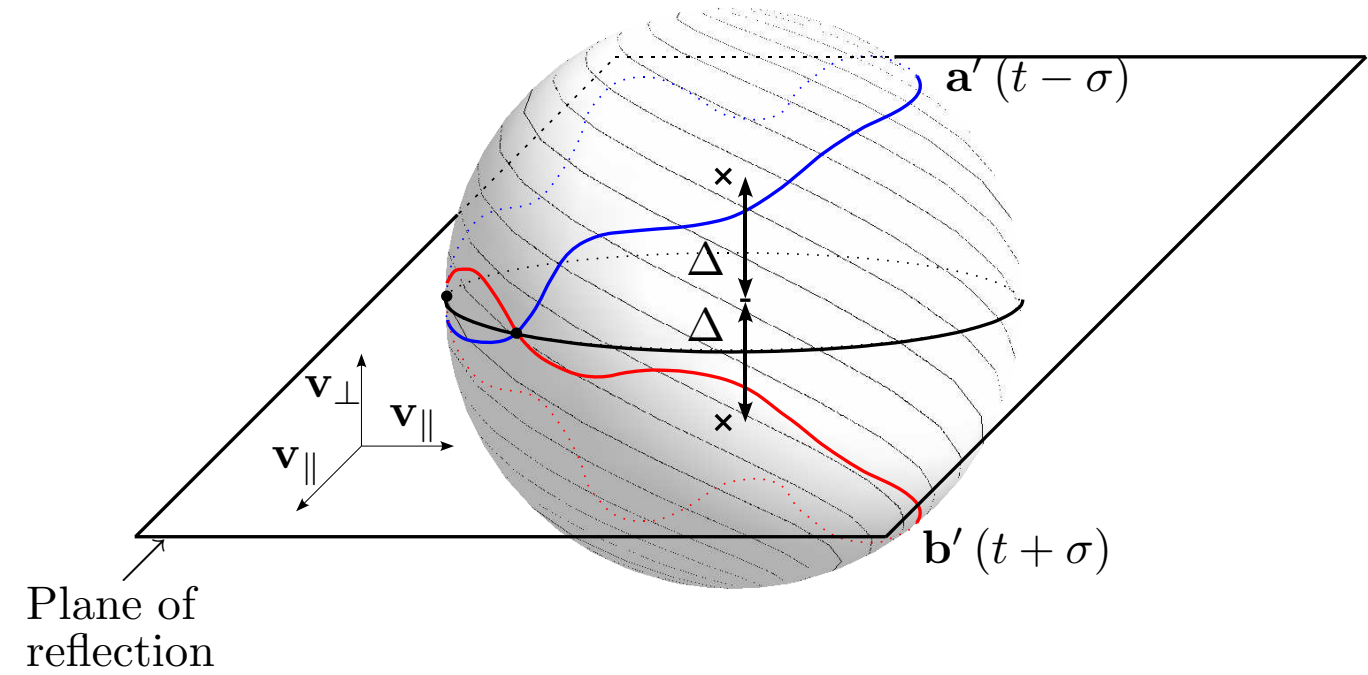

FIGURE 1. The solution for a Cosmic Superstring, governed by the DBI action, ending on two parallel D2-branes is given in terms of its right and left movers: a and $\mathbf{b}$. The boundary conditions imply that their total derivatives must be closed curves on the unit sphere, that are reflections of each other, with a 'center of mass' given by the inter-brane separation. When these curves intersect, the Cosmic Superstring will have a cusp. Note: $\mathbf{v}$ is an arbitrary vector that has been decomposed into components parallel $\left(v_{\|}\right)$and perpendicular $\left(v_{\perp}\right)$ to the branes.

these constraints are compatible with the equations of motion, which are,

$$
\ddot{\mathbf{x}}+\mathbf{x}^{\prime \prime}=0, \quad \dot{P}=P^{\prime}=0,
$$

where

$$
P \equiv \frac{\partial \mathscr{L}}{\partial F_{\tau \sigma}}=\frac{\lambda^{2} \mu F_{\tau \sigma}}{\sqrt{-x^{\prime 2} \dot{x}^{2}-\lambda^{2} F_{\tau \sigma}^{2}}}
$$

is the canonical momenta of the electromagnetic field.

Because the world sheet electromagnetic field is non-dynamical, the dynamics of a Cosmic Superstring are exactly as in the standard case. The difference arise because of the possibility of a Cosmic Superstring ending, either at a junction or on a D-brane. One can show [1] that for the case of a Cosmic Superstring ending of two parallel Dn-brane the left and right movers have the following properties,

$$
\begin{aligned}
\left|\mathbf{a}^{\prime}\right| & =\left|\mathbf{b}^{\prime}\right|=1, \\
\mathbf{a}^{\prime}(\xi) & =\mathbf{a}^{\prime}(\xi), \\
\mathbf{b}^{\prime}(\xi) & =\mathbf{b}^{\prime}(\xi), \\
\mathbf{a}_{\|}^{\prime}(\xi) & =\mathbf{b}_{\|}^{\prime}(\xi), \\
\mathbf{a}_{\perp}^{\prime}(\xi) & =-\mathbf{b}_{\perp}^{\prime}(\xi), \\
\frac{1}{2} \int_{-L}^{L} \mathbf{a}^{\prime}(\xi) \mathrm{d} \xi & =\Delta, \\
\frac{1}{2} \int_{-L}^{L} \mathbf{b}^{\prime}(\xi) \mathrm{d} \xi & =-\Delta,
\end{aligned}
$$

where $(\perp, \|)$ indicate respectively the directions perpendicular and parallel to the end branes and $\Delta$ is a vector stretching between the two branes. From the first three of these conditions we can see that, just as in previous case, we can visualize the solutions as closed curves on the unit sphere. However the fourth and fifth conditions tell us that the curves giving $\mathbf{a}^{\prime}$ and $\mathbf{b}^{\prime}$ are no longer independent, instead they are reflections of each other through a plane parallel to the branes. Finally the sixth and seventh conditions show that the 'center of mass' of these closed curves is not zero, but instead depends on the inter-brane separation. 


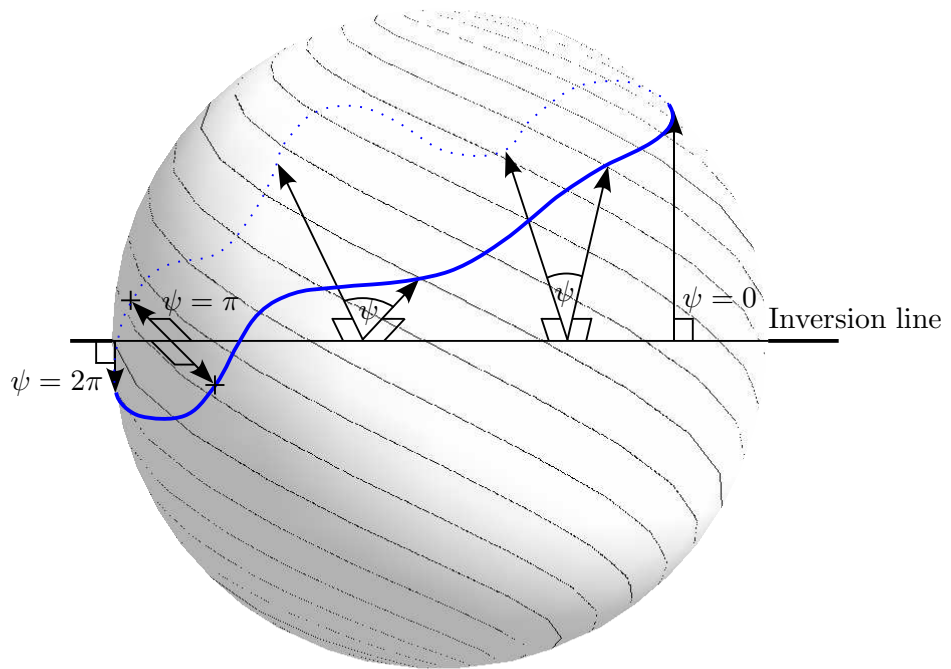

FIGURE 2. For the case of a DBI string ending on a pair of parallel D1-branes, one needs to decide if a closed curve, reflected through a line, will intersect itself. In the case of the reflection line being encircled by the closed curve, there will be such an intersection. This can be seen by considering the pair of vectors that are perpendicular to the reflection line and end on the closed curve. By continuity there must be at least one point at which the angle between these vectors $\psi=\pi$ and hence at least one intersection between this closed curve and its reflection.

The easiest case to consider is a DBI string ending of a pair of D2-branes. This situation is depicted in Figure 1 It is clear that if $\mathbf{a}^{\prime}$ (or $\mathbf{b}^{\prime}$ ) ever crosses the plane of reflection, then the two curves will intersect and the Cosmic Superstring will have a cusp. In the limit that the branes are separated by $L$, the coordinate length of the string, these closed curves become confined to the the points on the sphere furthest from the plane of reflection. Thus they will not intersect each other. However, when the branes are close together (i.e. when $\Delta \approx 0$ ), we can again see that intersections (and hence cusps) will be generic. A quantitative analysis of what constitutes 'close' in this context is work in progress, however the result that it is possible to get cusps on non-periodic Cosmic Superstring is robust.

The more interesting case for cosmology is that of a DBI string ending on a D1-brane, since this is a step towards modeling a string ending on a three string junction. This can also be visualized as a pair of closed curves on the unit sphere, however in order to demonstrate that the reflection of a closed curve though a line, will intersect itself, one has to consider some, essentially, topological arguments. In particular, consider the two vectors that are perpendicular to the reflection line and end on the closed curve. If the reflection line is encircled by the curve, then the angle between these two vectors is 0 at one extreme and $2 \pi$ at the other (see Figure 2). By continuity, the must be a point at which the angle between the vectors in $\pi$ and thus at least one pair of points which are mapped into each other by the reflection.

It fact it can be shown [1], that in approximately half of all possible cases such intersections will occur, at least whilst the end branes are close together. Thus in a significant proportion of the time, one would expect to have cusps on a Cosmic Superstring ending on a pair of D1-strings.

\subsection{Three string junctions}

In the previous section it was shown that it is possible to have cusps on Cosmic Superstrings that end on D-branes. Here we want to consider the case of a three string junction, formed after the collision between an F- and a D-string, which combine to produce a bound state FD-string [14]. The DBI action for such a junction, again working in the conformal gauge, is given by [13, 15],

$$
\begin{gathered}
S=-\sum_{i} \mu_{i} \int \mathrm{d} \tau \int_{0}^{L_{i}(\tau)} \mathrm{d} \sigma \sqrt{-x_{i}^{\prime 2} \dot{x}_{i}^{2}-\lambda\left(F_{\tau \sigma}^{i}\right)^{2}} \\
+\sum_{i} \int \mathrm{d} \tau\left\{\mathbf{f}_{i}(\tau) \cdot\left[x_{i}\left(\tau, L_{i}(\tau)\right)-\bar{x}(\tau)\right]+g_{i}\left[A_{\tau}^{i}\left(\tau, L_{i}(\tau)\right)+\dot{L}_{i} A_{\sigma}^{i}\left(\tau, L_{i}(\tau)\right)-\bar{A}(\tau)\right]\right\},
\end{gathered}
$$


where $\mu_{i}$ is the tension of the $i^{\text {th }}$-string, and $A_{\alpha}^{i}$ and $F_{\alpha \beta}^{i}$ are the gauge field and gauge field strengths respectively on the $i^{\text {th }}$-string. The Lagrange multipliers $\mathbf{f}_{i}(\tau)$ constrain the strings to meet at the junction (with world line $\bar{x}(\tau)$ ), whilst the Lagrange multipliers $g_{i}(\tau)$ impose to the component of each string's gauge field that is tangential to the junction world-line to coincide with the junction gauge field $\bar{A}(\tau)$. Note that, just as for cusps, the presence of a junction breaks the thin string limit (still present in the DBI-action), however numerical calculations show that the above action is a good approximation to the dynamics of such systems [16].

The equations of motion for each string in this action is the same as that of a single DBI-string (Eqs. (8)), however the boundary condition at the junction end (which we fix to be at the $\sigma_{i}=L_{i}(t)$ end of the strings) is given by the variation of Eq. (11) with respect to the Lagrange multipliers and with respect to $\bar{x}(\tau)$ and $\bar{A}(\tau)$. This gives the following set of conditions:

$$
x_{i}\left(\tau, L_{i}(\tau)\right)=\bar{x}(\tau)
$$

and

$$
\begin{gathered}
A_{\tau}^{i}\left(\tau, L_{i}(\tau)\right)+\dot{L}_{i} A_{\sigma}^{i}\left(\tau, L_{i}(\tau)\right)=\bar{A}(\tau) . \\
\sum_{i} \bar{\mu}_{i}\left(x_{i}^{\mu \prime}+\dot{L}_{i} \dot{x}_{i}^{\mu}\right)=0
\end{gathered}
$$

and

$$
\sum_{i} p_{i}=0
$$

where the effective string tension $\bar{\mu}_{i}$ is a combination of the tension and the electromagnetic field strength on the $i^{\text {th }}$ string. Note that as in the previous cases it is possible to fix the temporal gauge condition $\left(\tau=x^{0}=t\right)$ and that we can no longer fix the coordinate length of each string to be a constant, instead we have $L_{i}(\tau)$.

These equations can be used, along with the equations of motion, to find [14, 1]

$$
\frac{\bar{\mu}_{1}}{\bar{\mu}_{1}+\bar{\mu}_{2}+\bar{\mu}_{3}}\left(1-\dot{L}_{i}\right)=\frac{M_{1}\left(1-c_{23}\right)}{M_{1}\left(1-c_{23}\right)+M_{2}\left(1-c_{13}\right)+M_{3}\left(1-c_{12}\right)},
$$

with cyclic permutations giving expressions for $\dot{L}_{2}$ and $\dot{L}_{3}$, where

$$
M_{1}=\bar{\mu}_{1}^{2}-\left(\bar{\mu}_{2}-\bar{\mu}_{3}\right)^{2},
$$

with cyclic permutations giving $M_{2}$ and $M_{3}$ and scalar products are abbreviated to

$$
c_{i j}=\mathbf{a}_{i}^{\prime}\left(t-L_{i}(t)\right) \cdot \mathbf{a}_{j}^{\prime}\left(t-L_{j}(t)\right) .
$$

In order to make progress with this, we restrict our attention to the specific case of an F-, D- and FD-string junction (until now, the equations are applicable to any three DBI strings). For this situation we have the following relationship between the effective tensions $\bar{\mu}_{i}$ :

$$
\bar{\mu}_{1}=1, \quad \bar{\mu}_{2}=\frac{1}{g_{\mathrm{s}}}, \quad \bar{\mu}_{3}=\sqrt{1+\frac{1}{g_{\mathrm{s}}^{2}}}=\frac{1}{g_{\mathrm{s}}}+\frac{g_{\mathrm{s}}}{2}+\mathscr{O}\left(g_{\mathrm{s}}^{3}\right),
$$

where $g_{\mathrm{s}}$ is the perturbative string coupling and we have taken the $1^{\text {st }}$ string to be the F-string, the $2^{\text {nd }}$ string to be the D-string and the $3^{\text {rd }}$ string to be the bound state FD-string. To leading order in $g_{\mathrm{s}}$, Eqs. (16) give,

$$
\begin{aligned}
\left(S_{23}-2 S_{13}-2 S_{12}\right) \mathbf{b}_{1}^{\prime} & =S_{23} \mathbf{a}_{1}^{\prime}-2 S_{13} \mathbf{a}_{2}^{\prime}-2 S_{12} \mathbf{a}_{3}^{\prime}, \\
\mathbf{b}_{2}^{\prime} & =\mathbf{a}_{3}^{\prime}, \\
\mathbf{b}_{3}^{\prime} & =\mathbf{a}_{2}^{\prime},
\end{aligned}
$$

and

$$
\dot{L}_{1}=1-\frac{S_{23}}{S_{12}+S_{13}}, \quad \dot{L}_{2}=\frac{S_{12}-S_{13}}{S_{12}+S_{13}}, \quad \dot{L}_{3}=\frac{S_{13}-S_{12}}{S_{12}+S_{13}},
$$

where $S_{i j}=\frac{1}{2}\left(1-c_{i j}\right)$ and the notation

$$
\mathbf{a}_{i}^{\prime}=\mathbf{a}_{i}^{\prime}\left(t-L_{i}(t)\right) \quad \text { and } \quad \mathbf{b}_{i}^{\prime}=\mathbf{b}_{i}^{\prime}\left(t+L_{i}(t)\right),
$$


has been used.

The physical interpretation of this is clear, Eqs. (21) and (22) show that the in-movers on (heavy) D-string become the out-movers on the (heavy) bound state FD-string and vice verse. Also, we have that $\dot{L}_{2}+\dot{L}_{3}=0$. Thus the Dand the FD-strings are essentially behaving as a single long string, entirely unaffected (to order $g_{\mathrm{s}}$ ) by the presence of the (light) F-string or the junction. Whilst the (light) F-string moves in the background of the heavy string, it does so without back-reacting on its motion, similar in many ways to the Born-Oppenheimer approximation in condense matter physics.

For the F-string the junction has exactly the same boundary conditions as a standard D1-brane (albeit a moving D1brane), which we have already seen in Section (2.1). There we considered only static branes as boundary conditions, however the extension to moving branes can be done. Indeed, because the presence of cusps is a topological feature, a general class of deformations to the static case can be considered and shown to leave the presence of cusps unaltered. In particular the additional rotation that follows the reflection considered in Section (2.1), that is implied by Eq. (20) belongs to such a class and hence does not affect the presence of cusps.

\section{CONCLUSIONS}

We have shown that for a three string junction formed from F-, D- and FD-strings, the heavy D- and FD-strings behave as a single string, whilst the F-string moves as though the junction were a standard Dirichlet boundary conditions. This has several important consequences for the evolution of Cosmic Superstrings.

Firstly, the fact that the collision of F- and D-strings and the formation of bound states does not alter (to order $g_{\mathrm{s}}$ ) the dynamics of the heavy D-strings, implies that scaling will still be reached in this sector of the theory. There has been some numerical evidence [17] that suggests that this is indeed the case (at least for the case of the non-Albelian model of Cosmic Superstrings). However it remains to been seen how accurate the $g_{\mathrm{s}} \rightarrow 0$ limit is for such a model, particularly on the motion of loops of the heavy string (which can and will still form). It is possible that as the number of junctions increase, and the number and type of bound states increase (FFD-, FFFD-strings etc.), the probability of forming a 'free' heavy loop drops to zero. In which case the subsequent motion of the loops would be correlated with that of the long (heavy) strings.

Secondly, during collisions between F- and D-strings, the F-string will break onto the D-string, forming a pair of junctions. For any F-string ending of two such junctions, we have demonstrated that it will contain cusps. This represents an entirely new energy loss mechanism for the system, which may have significant consequences for the final scaling ratios (if scaling is indeed reached) of the energy in each sector. In addition, these cusps represent a way of distinguishing Cosmic Superstrings from standard Cosmic Strings, if they can be discriminated from the cusps present on loops of string. As mentioned in Section (1), the radiation produced by such cusps could, in principle, excite all possible String theory modes, making them a possible direct probe of string theory as well as Cosmic Superstrings.

Whilst we have shown that cusps are likely to be important features of strings ending on junctions, a quantitative analysis of the relative importance of loops and junctions, in the formation of cusps is still needed. In addition, the issue of small scale structure and its effect of the presence of cusps needs to clarified. In fact in standard Cosmic Strings, small scale kinks are expected to form at every collision event, however in the case of Cosmic Superstrings, many of these collisions will form junctions. This would seem to suggest that cusps are even more likely on Cosmic Superstrings, compared to standard field theoretic strings, however one would need to confirm this qualitative expectation with rigorous numerical or analytical work.

\section{ACKNOWLEDGMENTS}

This article is based on the longer and more detailed paper written with Anne-Christine Davis, Senthooran Rajamanoharan, and Mairi Sakellariadou [1].

This work was supported in part by the NSF grant PHY0854743, The George A. and Margaret M. Downsbrough Endowment and the Eberly research funds of Penn State.

\section{REFERENCES}

1. A.-C. Davis, W. Nelson, S. Rajamanoharan, and M. Sakellariadou, JCAP 0811, 022 (2008), 0809.2263 
2. N. Bevis, and P. M. Saffin, Phys. Rev. D78, 023503 (2008), 0804.0200

3. M. Sakellariadou (2009), 0902.0569

4. A. Vilenkin, and E. P. S. Shellard, Cosmic Strings and other topological defects, Cambridge University Press, 1994.

5. $\quad$ R. H. Brandenberger, Nucl. Phys. B293, 812 (1987).

6. K. D. Olum, and J. J. Blanco-Pillado, Phys. Rev. D60, 023503 (1999), gr-qc/9812040

7. X. Siemens, et al., Phys. Rev. D73, 105001 (2006), gr-qc/0603115

8. X. Siemens, V. Mandic, and J. Creighton, Phys. Rev. Lett. 98, 111101 (2007), astro-ph/0610920

9. C. D. Johnson, D-Branes, Camridge University Press, 2003.

10. E. J. Copeland, R. C. Myers, and J. Polchinski, JHEP 06, 013 (2004), hep-th/0312067

11. N. Barnaby, A. Berndsen, J. M. Cline, and H. Stoica, JHEP 06, 075 (2005), hep-th/ 0412095.

12. T. Damour, and A. Vilenkin, Phys. Rev. Lett. 78, 2288-2291 (1997), gr-qC/9610005

13. E. J. Copeland, H. Firouzjahi, T. W. B. Kibble, and D. A. Steer, Phys. Rev. D77, 063521 (2008), 0712.0808.

14. E. J. Copeland, T. W. B. Kibble, and D. A. Steer, Phys. Rev. Lett. 97, 021602 (2006), hep-th/0601153.

15. E. J. Copeland, T. W. B. Kibble, and D. A. Steer, Phys. Rev. D75, 065024 (2007), hep-th/ 0611243

16. P. Salmi, A. Achucarro, E. J. Copeland, T. W. B. Kibble, R. de Putter, and D. A. Steer, Phys. Rev. D77, 041701 (2008), 0712.1204

17. J. Urrestilla, and A. Vilenkin, JHEP 02, 037 (2008), 0712.1146. 